\title{
Evaluation of image quality in chest radiographs
}

\author{
Chand R B, Thapa N, Paudel S, Pokharel G B, Joshi B R, Pant D K
}

Department of Radiology and Imaging, Tribhuvan University Teaching Hospital, Maharajgunj

Corresponding address: Ram Bahadur Chand, Department of Radiology,Tribhuvan University Teaching Hospital, Maharajgunj,Kathmandu, Nepal,

Email: rbchand2003@yahoo.co.in

\begin{abstract}
Introduction: Conventional chest radiography is a proven and a useful procedure for imaging of the main airways, lungs, mediastinum, heart, pleura and chest wall. The basic view is PosteroAnterior (PA) of chest in upright position. The role and importance of quality of radiograph is to help the radiologists and the clinicians in the diagnosis of diseases and its management. Thus it is important to show entire anatomical structure, respiration accuracy by counting 6 anterior ribs/ 10 posterior ribs, adequate penetration to see the lower thoracic intervertebral discs through the heart and scapula out of lung fields. The study is an attempt to quantify the quality of chest radiographs by evaluating the quality of depiction of the anatomical and physical details.

Methods: A cross sectional study was carried out from June to September, 2012 in Tribhuvan University, Teaching Hospital, Maharajgunj. Chest radiographs were collected in the scheduled examination days and evaluated by under the guidance of Radiologist. Five image criteria i.e. anatomical coverage, adequate inspiration, adequate penetration, rotation and scapula out of lung fields were categorized and descriptive statistics were calculated using SPSS version 11.5 software.

Results: A total 1101 chest radiographs were collected from the radiology department for evaluation. Among them about 52.3\% chest radiographs met all image criteria but remaining $47.7 \%$ did not meet these criteria due to inadequate arrested inspiration (34.8\%), inadequate penetration $(24 \%)$, rotation $(21.8 \%)$, scapula not out of lungs field $(14.7 \%)$ and no anatomical coverage $(3.8 \%)$.

Conclusions: The results identified that performing good quality chest radiograph according to European guidelines is difficult. To a great extent the quality of chest radiographs depends upon skill of radiographer, equipment condition (x-ray machine, CR reader) and co-operation of patients. This is always challenging for radiographer and technologist.
\end{abstract}

Key words: Evaluation, image quality, chest radiographs

\section{Introduction}

Despite a small but increasing hazard of diagnostic $\mathrm{x}$ - ray to human beings ${ }^{1,2}$, studies aimed at achieving low patient doses with sufficient image quality have to be continuous to be area of research interest. The relationship between the quality of radiographic image and dose to patient is known to depend on the performance characteristics of the $\mathrm{x}$ - ray equipment, patients shape and size, type of image receptors, radiographic techniques, viewing condition as well as staff experience. ${ }^{3}$

The protocols that objectively establish these relationships are however, few worldwide and inexistent in many countries. Recently, the European Union has published a set of objective guidelines for good radiographic techniques and corresponding the level of image quality. ${ }^{4}$ The guideline has proved to be useful tool to unify the practice in Europe.

For the film critique of the chest, diagnostic standard for imaging is dependent not only on routine imaging criteria, but also on clinical information provided by the ordering physician. In this module we will explore some common reasons for taking a routine chest $\mathrm{x}$-ray. Keep in mind that while the diagnostic criteria for each view will be stated, the 
actual diagnostic criteria must also include proper exposure to demonstrate the requested diagnosis. This review of chest imaging will include pathology considerations since different types of pathology require change in exposure technique relative to normal lung tissue. For the good quality chest radiography coverage of whole lung fields from apex to dome of diaphragm, adequate arrested inspiration, adequate penetration, no rotation and scapula out of lungs field are required. ${ }^{5,6}$

\section{Methods}

The cross sectional study was conducted from June to September, 2012 at Tribhuvan University, Teaching Hospital, Kathmandu, Nepal. A total 1101 cases of chest radiographs were collected and these radiographs were performed in upright erect position by using Shimadzu $\mathrm{x}$-ray machine with capacity $150 \mathrm{kV}$ and $700 \mathrm{~mA}$. The $\mathrm{x}$-rays were performed in $\mathrm{kV}$ ranges from 90-110 and mAs ranges 15-60 using Computed Radiography image receptor (AGFA Company) of standard speed. The size of image receptor was 14"x14" with use of vertical stand. These X-rays were processed in AGFA CR 30 readers. Those patients who had thoracic deformities such as pectus excavatum, scoliosis and kyphosis were excluded and age group of patient ranged from 15-60 years in both sexes. All radiographs were reviewed by radiographers with the help of radiologist. The finding of each case was recorded in the Proforma sheet each day.

The qualities of all collected chest radiographs were evaluated under five criteria:

1. Anatomical coverage (include entire lung fields from apex to dome of diaphragm)

2. Arrested respiration (at least 6 anterior ribs and 10 posterior ribs above the right dome of diaphragm)

3. Adequate penetration (slightly visible lower intervertebral disc below T9)

4. No rotation (equidistant between spine of vertebra and sterno-clavicular joints) and

5. Scapula out of lung fields (medial border of scapula out of lung)

Each criterion was classified into yes or no category and descriptive statistics were calculated using SPSS version-11.5 software.

\section{Results}

Evaluation of image quality of collected chest radiographs in this study showed different values based on standard image criteria (categorized into anatomical coverage, arrested inspiration, adequate penetration, no rotation, scapula out of lungs field). These categories of standard image criteria were estimated (Table. 1).

Table 1: Evaluation of Image Quality

\begin{tabular}{|lllll}
\hline S.N Criteria & Yes & No & Total \\
\hline 1 & Anatomical & 1059 & $42(3.8)$ & $1101(100)$ \\
& coverage & $(96.2)^{*}$ & & \\
\hline 2 & Arrested & & & \\
\hline & inspiration & $718(65.2)$ & $383(34.8)$ & $1101(100)$ \\
\hline 3 & Adequate & & & \\
\hline & penetration & $837(76)$ & $264(24)$ & $1101(100)$ \\
\hline 4 & No rotation & $861(78.2)$ & $240(21.8)$ & $1101(! 00)$ \\
\hline 5 & Scapula out & & & \\
\hline & of lung fields & $939(85.3)$ & $162(14.7)$ & $1101(100)$
\end{tabular}

*Parenthesis indicates percentage

All correct image criteria

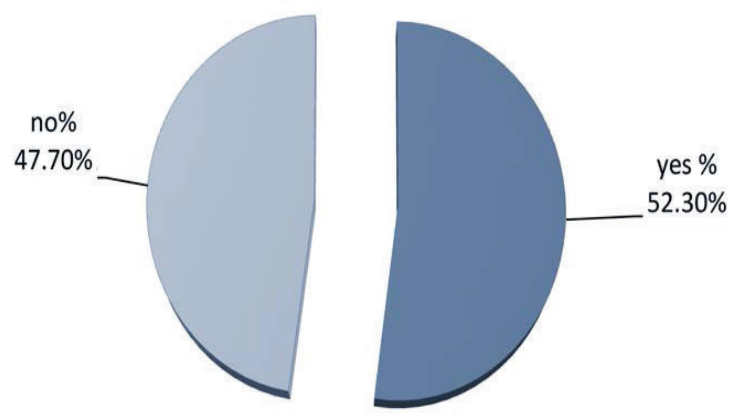

Figure1 Categorization of all correct images

On the basis of anatomical coverage, the study showed that $96.2 \%$ radiographs were included all lung fields and $3.8 \%$ were not. In term of arrested inspiration, the study showed $65.2 \%$ radiographs included adequate arrested inspiration and $34.8 \%$ poor inspiration. On the basis of adequate penetration, study showed only $76 \%$ radiographs were correct and 34\% incorrect. In the category of no rotation, $78.2 \%$ radiographs did not show rotation and $21.8 \%$ showed rotation. Evaluation of scapula out of lung fields category showed $85.3 \%$ radiographs were correct and $14.7 \%$ incorrect. All correct criteria evaluation showed 
$52.3 \%$ radiographs were correct and $47.7 \%$ were incorrect as shown in figure 1.

\section{Discussion}

According to European Guidelines for image quality in chest radiograph4 the chest radiograph should include both lung apices and both costophrenic angles. There should be equidistant between the spine of vertebra and medial ends of clavicles. There should be at least 6 th anterior ribs above diaphragm for full inspiration. There should be definition of lower intervertebral disc below T9 for adequate penetration. The medial border of scapula should be out of lungs field for proper position of patient.

In the case of evaluation of anatomical criteria for chest, the error was due to the improper position ${ }^{7,8}$, small size of image receptor and patient shift in between positioning and exposure. In evaluation of arrested inspiration in chest radiograph, the error was due to inappropriate instruction given to patients by radiographer or due to the obese patients. In terms of adequate penetration ${ }^{7,8}$, error was due to the under exposure given to patients. In rotation error because of the improper position and in scapula out of lungs field error was because of improper position of arm of patients.

In overall correct criteria only $52.3 \%$ met all of above five criteria. But remaining about $47.8 \%$ was incorrect due to either of one of above or more than two reasons. Incorrect chest radiographs were unacceptable for reporting and required repetition. Due to repetition there was increased radiation dose to patients, increased workload and economic loss for department. The errors in anatomical coverage, arrested respiration, adequate penetration and massive rotation altered the diagnosis. These errors were too high for acceptance.

Only the $52.3 \%$ chest radiographs met the major European criteria for chest PA radiograph. An attempt had been made to identify the cause of poor quality of radiographs, where attention must be focused to improve the technique and positioning of patient. The main faults identified were mid arrested respiration, inadequate penetration, rotation of patients, non visualization of whole lung fields. Among them major fault was due to inadequate arrested inspiration during chest $\mathrm{x}$-ray examination and minor cause was due to anatomical cut-off.

Evaluation of specific features in chest radiograph was found to be feasible and practicable for routine use. While it is normally easy to say that one image was better than the other. It was difficult to define which feature or the other influence this decision. It was clear from the study that all the criteria which tend different aspects; correlate fairly well with the quality of radiographs.

\section{Conclusion}

To a great extent the quality of chest radiographs depends upon skill of radiographer, equipment condition (x-ray machine, $\mathrm{CR}$ reader) and co-operation of patients. We should always keep in mind to provide standard quality radiographs and do accordingly. This is always challenging for radiographer and technologist.

Conflict of interest: The authors declare that they have no conflict of interests.

\section{References}

1. International Commission on Radiology Protection, 1990 recommendation of the International Commission on Radiology Protection, ICRP Publication. 1991; 60Ann.ICRP21 (1-3).

2. United Nations Scientific Committee on the Effect of Atomic Radiation (UNSCEAR) source and effects of ionizing radiation (Newyork: United Nations) (2000).

3. Stieve EE, HagemannG and Sterder H. Relationship between medical requirements and technical parameters of good imaging performance and acceptable dose radiation. Prot.Dosin.1993; 19(1/3),3-18.

4. European Commission-European Guidelines on quality criteria for diagnostic radiographic images, 1996; Brussels, Europeans commission.

5. Moller, Pocket Atlas of Radiographic Anatomy, 2nd edition; thieme, 2000; 195.

6. Karen Duncan, Rowena Warwick. Adapted anatomical image criteria for PA chest Radiography. ACR, 2011.

7. Merrill's Atlas of Radiographic positioning and Radiological Procedure. 11th edition, volume 1; Mosby, 2007; 530

8. Sr. Lisanto F.C.C., M. Muthunel, Sreekumar, Quality assurance in chest radiographs, Roentgen Technology, $2005 ; 2 ; 30-35$. 